\title{
CSS VERSUS LARGE SIZE SOURCES
}

\author{
R. FANTI ${ }^{1}$ AND R. E. SPENCER ${ }^{2}$ \\ 1 Istituto di Radioastronomia del CNR, Bologna \\ 2 Nuffield Radio Astronomy Laboratories, \\ University of Manchester, Jodrell Bank
}

\section{Introduction}

A large fraction of the sources in flux density limited radio samples have angular sizes $<2$ arcsec (and hence projected linear sizes $\leq 10-15 \mathrm{kpc}$ for $H_{0}=100 \mathrm{Km} /(\sec \mathrm{Mpc})$, and steep $\left(\alpha>0.5, \mathrm{~S} \alpha \nu^{-\alpha}\right)$ high frequency spectra (Kapahi, 1981; Peacock and Wall 1982). The proportion of these Compact Steep-spectrum Sources (CSSs) is high (15-30\% depending on the selection frequency) amongst distant $(z>0.2)$ radio sources of high power, both galaxies and quasars. We include in this class the $\mathrm{GHz}$ Peaked Spectrum Radio Sources (GPS), sub-kpc objects whose radio spectra are peaked at $\mathrm{GHz}$ frequencies (see, e.g., O'Dea et al, 1991).

Statistical analyses have shown that the majority of CSSs cannot be larger sized sources foreshortened by projection effects (Fanti et al., 1990) so that their radio emission actually originates on sub-galactic scales.

What are the connections between CSSs and larger sized radio sources? Are CSSs part of an evolutionary sequence, in which they represent the early stage (youth scenario; e.g. Phillips and Mutel, 1982; Carvahlo, 1985)? Or are they instead a separate class of objects, where unusual conditions in the interstellar medium, such as higher density and/or turbulence, inhibit the radio source from growing to large dimensions (frustration scenario; e.g. van Breugel et al., 1984)?

Well studied samples of CSSs are those from the 3CR catalogue, selected at $178 \mathrm{MHz}$, and from the Peacock and Wall catalogue, selected at $2.7 \mathrm{GHZ}$ (Fanti et al., 1995 and references therein; Sanghera et al., 1995). A new sample of CSS has been produced as a result of VLBI surveys on sources selected 
at $5 \mathrm{GHz}$ (hereafter called CJ, see Readhead 1995a, and references therein). The frequency of selection is important since high frequency samples tend to include more sources with self-absorption low frequency turnovers. For example the CJ sample contains objects whose peak frequencies are larger than those in the PW sample and with smaller angular sizes.

\section{Radio Morphology}

The core fractional luminosities of CSSs are similar to those of large size radio sources of similar power, for both radio galaxies and quasars (median value $\approx 3 \%$ for quasars and $\leq \mathbf{0 . 4 \%}$ for radio galaxies). Boosting is therefore similar in the CSSs and larger radio sources and hence the CSSs are believed to have similar orientations in the sky to the large size double sources.

Double-lobed structure in CSSs is common in both quasars $(\geq 50 \%)$ and galaxies $(\approx 90 \%)$. By double-lobed structure we mean that extended radio emission, excluding jet(s), is seen on both sides of the centre of activity, identified by the radio core. In fact radio cores have been detected in only $70 \%$ of quasars and $40 \%$ of radio galaxies. Nevertheless we are confident, as indicated by the similarity of morphologies, that we can correctly classify those with truly symmetric structure even when the core is undetected

In order to emphasize the symmetry of the radio structure, we follow Readhead (1995a), using the name CSO's (Compact Symmetric Objects) for double lobed CSSs of small linear size ( $\leq 0.5 K p c$, mostly from the CJ sample), and MSO's (Medium-size Symmetric Objects) for the two lobed CSS's with sizes $\geq 0.5 K p c$ (mostly from the PW and 3CR samples).

These sources seem to be down-scaled versions of the large size doubles. However, asymmetries in lobe flux density ratio, or in arm ratio, or both, are more pronounced (Fanti et al., 1990; Sanghera et al., 1995).

A second minor group of CSSs is composed of objects with either complex morphology or strongly asymmetric emission with respect to the core. The majority of them are quasars with most of the luminosity being contributed by the jet (Spencer 1994). These distortions may be due to amplification by projection of small intrinsic bending, but the weakness of the core requires the line of sight to be larger for the core than the jet.

Bright jets are found also among the MSOs, mainly in quasars and less frequently in radio galaxies. They are certainly more prominent than in the large size doubles. The jet luminosity could be due not only to boosting, but also to interactions of the jet with the external medium. Interactions can convert the kinetic energy carried by the jet to internal energy and then to radiation, causing the jet to brighten.

In conclusion, CSSs seem to contain two classes of objects. One class is characterized by a symmetric structure and mimics the characteristics 
of the large size doubles. It is tempting to identify these sources with the progenitors of the large doubles.

The other class, contains objects with very distorted structure and often with very bright jets. These could be cases of "frustrated" radio sources. The class is dominated by quasars but it is unclear if this is because of an intrinsic property of quasars.

\section{Physical parameters and source evolution}

For a source with average luminosity in the sample, the equipartition pressure in the lobes can be parameterized as $p_{e q} \approx 5 \times 10^{-8} D_{k p c}^{-2}$ dyne $\mathrm{cm}^{-2}$, where $D_{k p c}$ is the half size of the source. We ask if, or under what conditions, a source with MSO properties remains confined or grows progressively larger with time.

Using simple one dimensional ram-pressure arguments, the advance speed of the lobes is determined by the equilibrium between the internal pressure and the ram-pressure of the external medium, $p_{i} \approx n_{\text {ext }} m_{p} v_{l}^{2}$ (where $n_{e x t}$ is the particle density of the external medium, $m_{p}$ is the proton mass and $v_{l}$ the lobe advance speed).

We assume an external density profile of the King type with central density $n_{o}$, core radius $r_{c}$ and asymptotic behaviour $n_{\text {ext }} \propto \operatorname{distance}^{-\beta}$, with $1.5 \leq \beta \leq 2$. If we take $n_{0} \approx 0.1 \mathrm{~cm}^{-3}$ and $r_{c}=2 \mathrm{kpc}$, then the time required by an average MSO to attain a size of $\approx 10 \mathrm{kpc}$ is $\approx 1.5 \times 10^{6}$ years. In order to keep the source small ( $\leq 15 \mathrm{Kpc}$ over $2 \times 10^{7}$ years) combinations of central density and core radius ranging from $n_{o} \approx 10 \mathrm{~cm}^{-3}$ and $r_{c}=$ $1 \mathrm{Kpc}$ to $n_{o} \approx 1 \mathrm{~cm}^{-3}$ and $r_{c} \approx 5 \mathrm{Kpc}$ are required.

If the medium is either hot $\left(10^{7} \mathrm{~K}\right)$ or tepid $\left(10^{4} \mathrm{~K}\right)$, we would expect high luminosities in X-rays or in emission lines, which have not been observed (Fanti et al., 1995). So we think it unlikely that the bulk of the CSOs and MSOs are frustrated radio sources (see also De Young, 1993).

\section{CSOs and MSOs as young radio sources}

If the CSOs and MSOs are young objects, with ages $\leq 10^{6}$ years, and the typical time scale of activity of the central engine is $\approx 2 \times 10^{7}$ years, then these objects should be a small fraction of the population of which they are the young phase. Since the MSOs comprise $\approx 30-40 \%$ of the high luminosity source population, there seems to be a contradiction. This difficulty is avoided if, during their evolution, radio sources either increase their velocity of expansion, or decrease in radio luminosity. These alternative possibilities have been discussed recently by Fanti et al. (1995), and Readhead et al. (1995 a,b). 
For the first scenario the "ram pressure" model requires an external density $n_{e} \propto r^{-3.3}$, and a rather high central density $\left(n_{o} \approx 70 \mathrm{~cm}^{-3}\right)$. These figures seem quite unrealistic.

The second scenario requires a luminosity $\propto$ size $e^{h}$ where $h \approx 0.4-0.5$ to be consistent with the observed size distribution. This also fits with the prediction of the continuous energy supply model derived from Scheuer (1974) and discussed by Baldwin (1982). In this model the radio luminosity decreases as the source grows, due to lobe expansion in the external medium. The decrease in luminosity required for CSOs/MSOs being young sources is obtained for $\beta \approx 1.5-2$.

\section{Conclusions}

We have distinguished between double lobed CSSs (for which we adopt the names CSOs/MSOs, following Readhead 1995a) and complex morphology or asymmetric jet dominated CSSs. We suggest that the first class contains the precursors of the large size double lobed radio sources. The required condition is that while they increase in size they undergo either an increase in their expansion velocity or a decrease in their luminosity. We think that the second possibility is the most realistic.

\section{References}

Baldwin, J., in Extragalactic Radio Sources, IAU Symp. 97, D.S. Heeschen and C.M. Wade, Reidel, 1982, 21

van Breugel, W.J.M., Miley, G.K., Heckman, T.A.,1984, Astron. J. 89, 5

Carvahlo J.C., 1985, Monthly Notices Roy. Astron. Soc. 215, 463

de Young, D.S., 1993, Astrophys.J. 402, 95

Fanti, R., Fanti, C., Schilizzi, R.T., Spencer, R.E., Nan Rendong, Parma, P., van Breugel, W.J.M., Venturi, T., 1990, Astron. Astrophys. 231, 333

Fanti, C., Fanti, R., Dallacasa D., Schilizzi, R.T., Spencer, R.E., Stanghellini C., 1995, Astron. Astrophys., 302,317

Kapahi V.K., 1981, Astron. Astrophys. Suppl. 43, 381

Mutel, R.L. and Phillips, R.B., in The impact of VLBI on Astrophysics and Geophysics, IAU Symp. 129, M.J. Reid and J.M. Moran, Reidel, 1988, 73

O'Dea, C.P., Baum, S.A., Stanghellini, C., 1991, Astrophys.J. 380, 66

Peacock J.A., Wall J.V., 1982, Monthly Notices Roy. Astron. Soc. 198, 843

Phillips T.J., Mutel R.L., 1982, Astron. Astrophys. 106, 21

Readhead A.C.S., 1995a, Quasars and AGN: High Resolution Imaging, Irvine 1995, eds. Cohen and Kellermann, Publications National Academy of Sciences, in press

Readhead A.C.S., Taylor G.B., Pearson T.J., Wilkinson P.N., 1995b, Astrophys. J, in press

Sanghera H.S., Saikia D.J., Ludke E., Spencer R.E., Foulsham P.A., Akujor C.E., Trioumis A.K., 1994, Astron. Astrophys. in press

Scheuer, P.A.G., 1974, Monthly Notices Roy. Astron. Soc. 166,513

Spencer R.E., 1994, in Compact Extragalactic Radio Sources, Proceedings of a workshop held at Socorro, eds. Zensus J.A. and Kellermann K.I., p. 35 